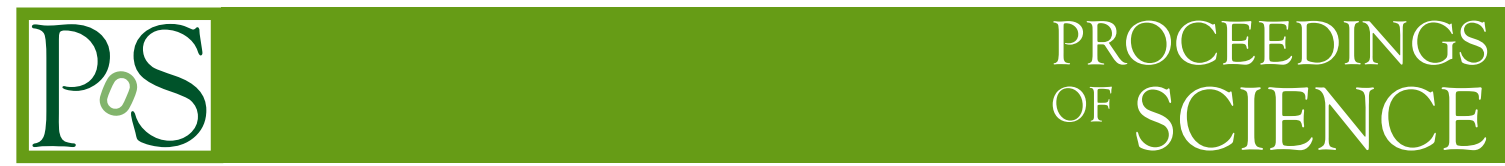

\title{
Long distance contribution to $K_{L}-K_{S}$ mass difference
}

\author{
Jianglei Yu* \\ Department of Physics, Columbia University, New York, NY 10025, USA \\ E-mail: jy2379@columbia.edu
}

\begin{abstract}
We present a method to non-perturbatively determine the long-distance contribution to the $K_{L^{-}}$ $K_{S}$ mass difference. The calculation is performed on $2+1$ flavor, domain wall fermion, $16^{3} \times 32$ configurations with a $421 \mathrm{MeV}$ pion mass and a kaon mass of $559 \mathrm{MeV}$. We include only currentcurrent operators and drop all disconnected diagrams in the calculation. The largest contribution comes from quadratically divergent, short distance lattice artifacts. This quadratic divergence is eliminated through the GIM mechanism by introducing a valence charm quark. The remaining short distance effects are then removed by using a RI/MOM technique which allows their exact replacement by the physical short distance part.
\end{abstract}

XXIX International Symposium on Lattice Field Theory

July 10-16 2011

Squaw Valley, Lake Tahoe, California

\footnotetext{
*Speaker.
} 


\section{Introduction}

The extremely small $K_{L}-K_{S}$ mass difference has been measured accurately decades ago. The origin of this difference is the $K^{0}-\bar{K}^{0}$ mixing via second order weak interactions. Conventionally, the mass difference is separated into a short-distance part and a long-distance part. While the short-distance contribution has been calculated to next-to-leading order [1], the long-distance contribution, which contributes around 30\% to the mass difference [2], can only be determined non-perturbatively. A year ago a lattice QCD method was suggested to compute the long-distance contribution [3]. This proceeding is the first numerical experiment using the new method.

\section{Second order correlator}

To compute the $K_{L}-K_{S}$ mass difference on a Euclidean space lattice, we can evaluate the timeintegrated second-order product over a time interval $\left[t_{a}, t_{b}\right]$ :

$$
\mathscr{A}=\frac{1}{2} \sum_{t_{1}=t_{a}}^{t_{b}} \sum_{t_{2}=t_{a}}^{t_{b}}\left\langle\bar{K}^{0}\left(t_{f}\right) H_{W}\left(t_{2}\right) H_{W}\left(t_{1}\right) K^{0 \dagger}\left(t_{1}\right)\right\rangle
$$

Here the kaon is created at $t_{i}$, the effective weak Hamiltonian acts twice within time interval $\left[t_{a}, t_{b}\right]$, and the outcome anti-kaon is annihilated at $t_{f}$. The amplitude is represented schematically in Figure 1 .

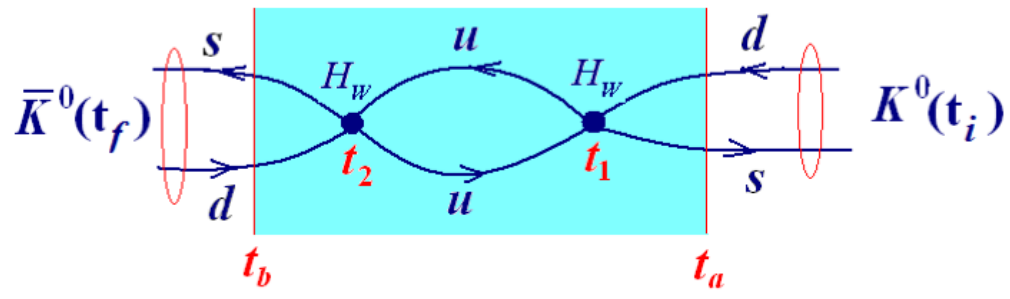

Figure 1: Example of the second order correlator $\mathscr{A}$ in Equation 2.1. Here the two four quark operators are integrated over the shadowed region.

If we assume that $t_{a}-t_{i}$ and $t_{f}-t_{b}$ are large enough for the interpolating operators to project onto kaon states, after inserting a complete set of intermediate states, Eq 2.1 becomes:

$$
\mathscr{A}=\left|Z_{K}\right|^{2} e^{-M_{K}\left(t_{f}-t_{i}\right)} \sum_{n} \frac{\left\langle\bar{K}^{0}\left|H_{W}\right| n\right\rangle\left\langle n\left|H_{W}\right| K^{0}\right\rangle}{\left(M_{K}-E_{n}\right)^{2}}\left\{e^{\left(M_{K}-M_{n}\right) T}-\left(M_{K}-M_{n}\right) T-1\right\}
$$

Here $T=t_{b}-t_{a}+1$ and $Z_{K}$ is the normalization factor of kaon interpolating operator. We assume that there is no intermediate state degenerate with kaon in this expression, which is true in this work. The term proportional to $T$ in Equation 2.2 gives the finite volume approximation to $K_{L}-K_{S}$ mass difference.

$$
\Delta M_{K}^{F V}=2 \sum_{n} \frac{\left\langle\bar{K}^{0}\left|H_{W}\right| n\right\rangle\left\langle n\left|H_{W}\right| K^{0}\right\rangle}{M_{K}-E_{n}}
$$

The dependence of the other terms in Equation 2.2 on $T$ can be classified into three categories: i). The exponential decreasing term, if $E_{n}>M_{K}$, these terms can be neglected when $T$ is sufficiently 
large; ii). Exponential increasing term, if $E_{n}<M_{K}$, these terms must be identified independently and subtracted from the result; iii) The term independent of $T$, which is trivial.

The full $\Delta S=1$ effective Hamiltonian consist of 7 independent four-quark operators [4], we include only the current-current operator $Q_{1}$ in this work.

$$
Q_{1}=\left(\bar{s}_{\alpha} d_{\alpha}\right)_{V-A}\left(\bar{u}_{\beta} u_{\beta}\right)_{V-A}
$$

The four different types of contractions are listed in Figure 2. In this work, we only compute type 1 and type 2 contractions and drop type 3 and type 4 contraction. Type 3 contraction is dropped because it is disconnected diagram. We need to compute extra random source propagators for type 4 contraction. So we also drop type 4 contraction in this first numerical experiment.

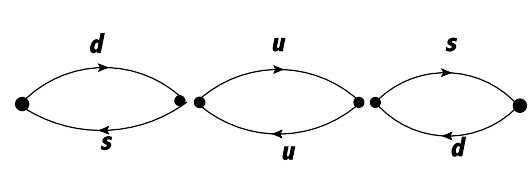

Type 1
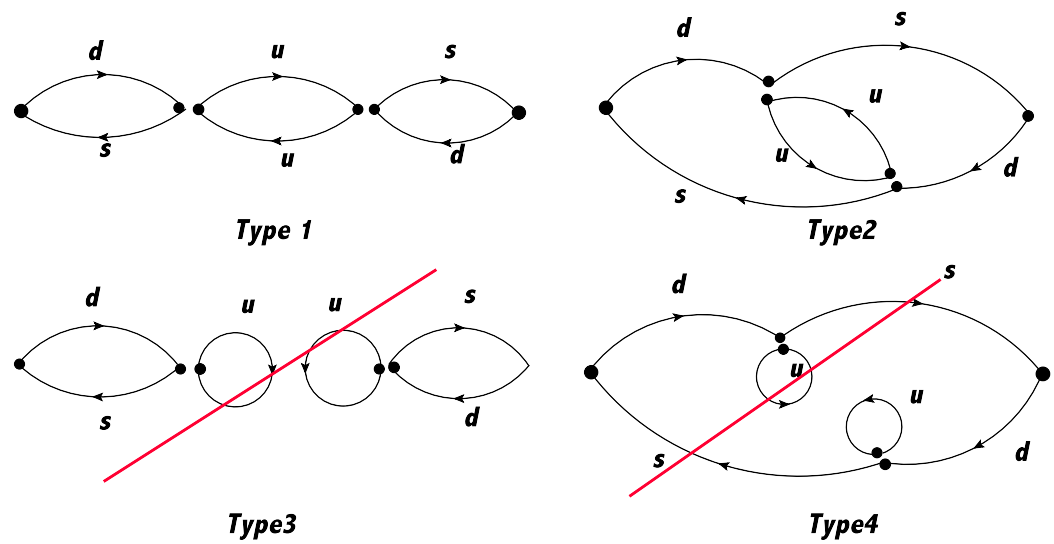

Figure 2: Four types of contractions in the 4-point correlator. Only type 1 and type 2 are included in the calculation, type 3 and type 4 are dropped.

As we mentioned before, we must identify the exponential increasing terms in Eq 2.2. These terms come from the intermediate states which are lighter than kaon, such states are $\pi^{0}$ state and vacuum state in this calculation. Since the disconnected diagrams are neglected, there will be no vacuum intermediate state. Then we must calculate $\left\langle\pi^{0}\left|Q_{1}\right| K^{0}\right\rangle$ and subtract the exponential increasing contribution from Equation 2.2. The contractions in this calculation are given in Figure 3. We drop type 2 to be consistent with 4-point correlator calculation.

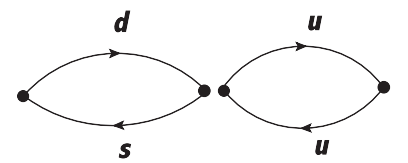

Type 1

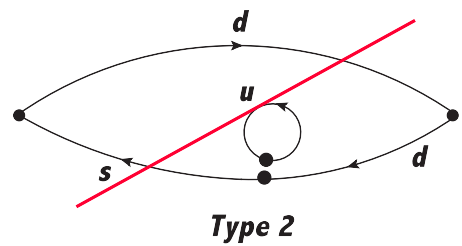

Type 2

Figure 3: Two types of contractions in $\left\langle\pi^{0}\left|Q_{1}\right| K^{0}\right\rangle$ 3-point correlator. Type 2 is dropped to be consistent with 4-point correlator in Figure 2.

\section{Short distance effect}

The computation was performed on a RBC/UKQCD ensemble with $N_{f}=2+1$ flavors, a $16^{2} \times 32 \times 16$ lattice volume, with dynamical domain wall fermions (DWF), the Iwasaki gauge 
action, $a^{-1}=1.73(3)$ Gev with a421 Mev pion mass and a $559 \mathrm{Mev}$ kaon mass. The two wallsource kaons are located at time slice $t_{i}=0$ and $t_{f}=27$. The two $\Delta S=1$ operator act within the time interval $[4,23]$. We analyzed 600 configurations separated by 10 Monte Carlo time units. The result is given in the first plot in Figure 4. The plot shows the integrated second order correlator as a function of the integration time interval $\mathrm{T}$. For each given integration time interval $\mathrm{T}$, we calculate the integrated correlator in Equation 2.2 between all possible time interval $\left[t_{a}, t_{a}+T-1\right]$ and take the average as the final result. The two curves in the plot are the results before and after the subtraction of the $\pi^{0}$ exponential term. The results have both a long-distance part and a shortdistance part. The short distance part means that the two $\Delta S=1$ operator are close to each other on the lattice. We expect the short distance part to be quadratically divergent because of the up quark loop in Figure 1. To get a detailed understanding of the short distance effect, we can introduce an artificial cut off, i.e., require the separation between two operators $\left.\left|x_{2}-x_{1}\right|\right\rangle r$. This cutoff will reduce the short distance effect and the long distance part will remain untouched. The other plot in Figure 4 shows the results with cutoff radius 5. We can see that the amplitude of the result is reduced substantially after introcuding the cutoff. And the contribution from $\pi^{0}$ intermediated state becomes visible. We can measure the mass difference at different cutoffs. From Equation 2.2, the mass difference is given by the coefficient of the linear term up to some factor when $\mathrm{T}$ is large. We choose to fit the slope of integrated correlator plot in the range $T \in[11,20]$. The mass differences are listed in Table 1. We can do a naive inverse quadratic fit for the mass differences at different cutoffs. The result is in Figure 5, which suggest that the short distance effect is quadratically divergent.

Table 1: Mass differences at different cutoff radius

\begin{tabular}{cccccc}
\hline Cutoff Radius & 1 & 2 & 3 & 4 & 5 \\
\hline$\Delta M_{K}$ & $0.3342(80)$ & $0.1533(30)$ & $0.0796(17)$ & $0.0560(15)$ & $0.0455(14)$ \\
\hline
\end{tabular}
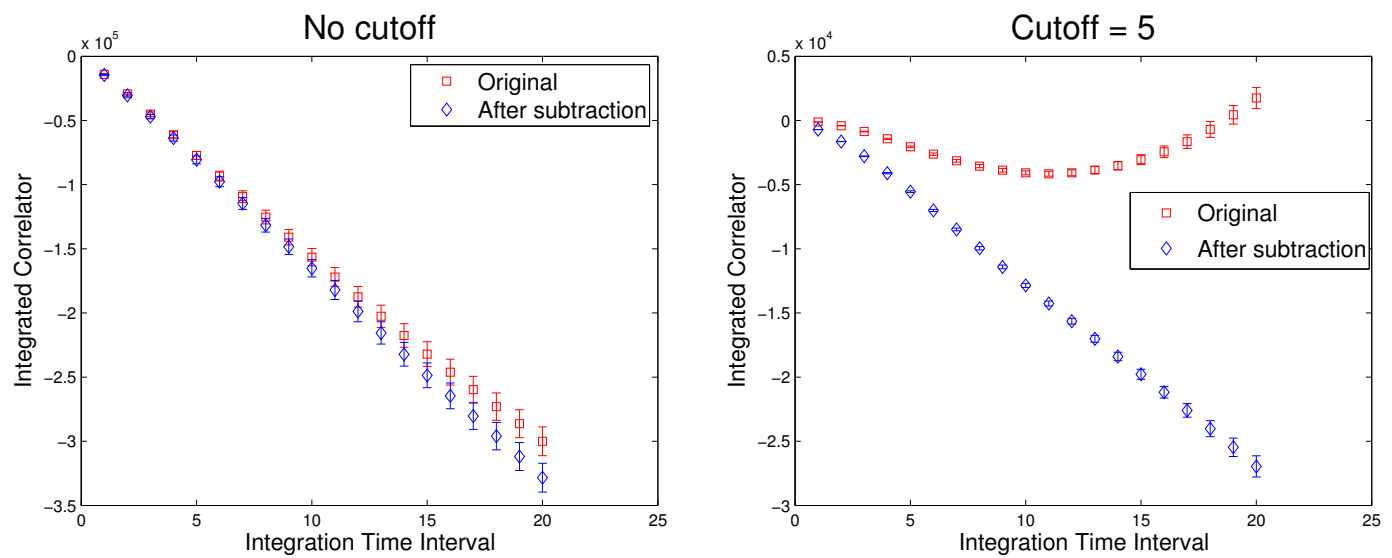

Figure 4: Integrated second order correlator as a function of integration time interval. The red and blue curve show the results before and after the subtraction of $\pi^{0}$ exponential term. The first plot is the result without any cutoff. The second plot shows the result with cutoffs radius 5 . 


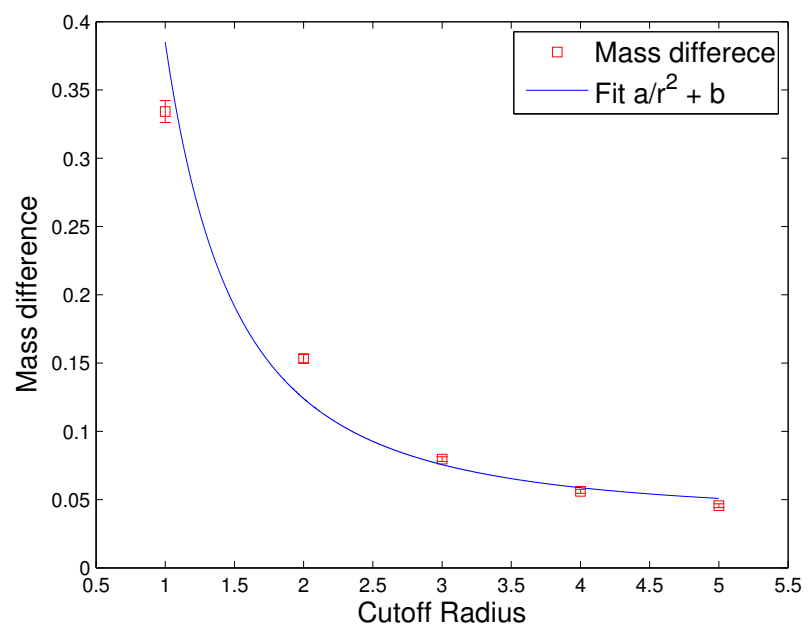

Figure 5: The mass differences at different cutoff radii, the blue cure is a naive, two-parameter quadratic fit.

\section{Charm quark and GIM}

In order to make the short distance more manageable, we introduce a valence charm quark to the calculation. Then GIM mechanism will reduce the divergency from quadratic into logarithmic. To implement this in the lattice calculation, we can replace all the up quark propagators in Figure 2 with the difference between up quark propagator and charm quark propagator. We use five difference valence charm quark mass ranging from $200 \mathrm{Mev}$ to $1000 \mathrm{Mev}$. The integrated correlators after GIM subtraction are plotted in Figure 6. The resulting mass difference $\Delta M_{K}$ for five difference valence charm quarks are listed in Table 2.

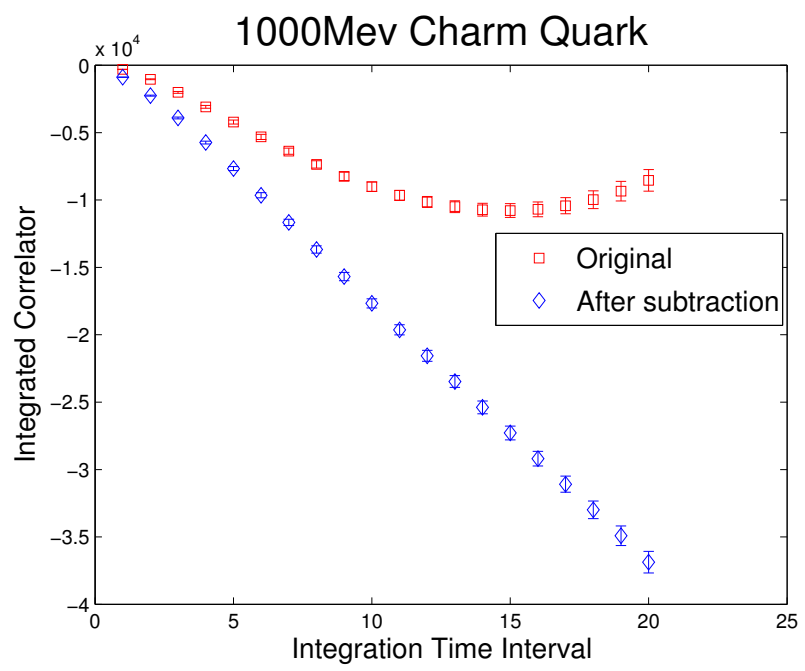

Figure 6: The integrated correlator after the inclusion of $1000 \mathrm{Mev}$ charm quark. The red and blue curves show the results before and after the subtraction of the $\pi^{0}$ exponential term 


\section{Remaining Short distance effect}

Even with the valence charm quark, there will still be some short distance lattice artifacts remaining. The short distance part in Equation 2.1 can be represented by the matrix element of a local four-quark operator.

$$
\begin{aligned}
\mathscr{A}_{S D} & =\frac{1}{2} \sum_{t=t_{a}}^{t_{b}}\left\langle\bar{K}^{0}\left(t_{f}\right) C(\mu) \mathscr{O}(t) K^{0 \dagger}\left(t_{i}\right)\right\rangle \\
& =\frac{1}{2}\left|Z_{K}\right|^{2} e^{-M_{K}\left(t_{f}-t_{i}\right)} C(\mu)\left\langle\bar{K}^{0}|\mathscr{O}| K^{0}\right\rangle T
\end{aligned}
$$

Here $T=t_{b}-t_{a}+1, \mathscr{O}=(\bar{s} d)_{V-A}(\bar{s} d)_{V-A}, C(\mu)$ is determined by the short distance part of the original second order amplitude and depends on the scale $\mu$ used in the definition of the operator $\mathscr{O}$. To identify $C(\mu)$ by using RI/SMOM techniques, we can evaluate off-shell, four quark, amputated Green's function for the two diagrams in Figure 7 at some large external momentum scale $\mu$. The
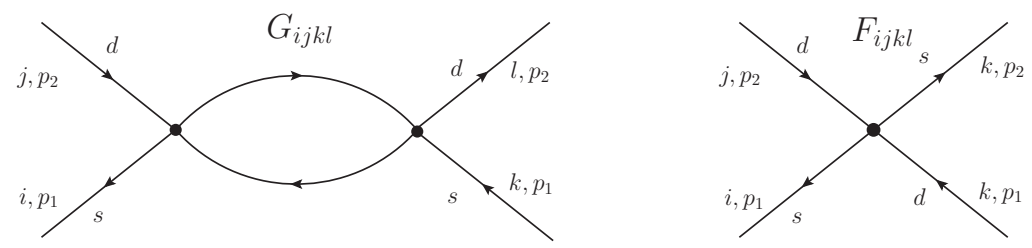

Figure 7: Off-shell, amputated four quark Green's functions. The left diagram is for two $\Delta S=1$ operators; the right diagram is for one $\Delta S=2$ operator.

external momentum satisfy $p_{1}^{2}=p_{2}^{2}=\left(p_{1}-p_{2}\right)^{2}=\mu^{2}$. Suppose the results for the two diagrams in Figure 7 are $G_{i j k l}$ and $F_{i j k l}$ respectively. Then we project the green functions onto the desired gamma structure $P_{i j k l}=\left(\left(1-\gamma^{5}\right) \gamma_{\mu}\right)_{j i}\left(\left(1-\gamma^{5}\right) \gamma_{\mu}\right)_{l k}$. The conversion factor is given by Equation 5.2.

$$
G(\mu)=G_{i j k l} P_{i j k l} \quad F(\mu)=F_{i j k l} P_{i j k l} \quad C(\mu)=\frac{G(\mu)}{F(\mu)}
$$

In Figure 8, the left plot shows the dependence of $C(\mu)$ on the momentum scale $\mu$ if we use a $1 \mathrm{GeV}$ valence charm quark. As we expected, $C(\mu)$ will become less significant as increasing $\mu$, because the difference between the charm and up quarks will decrease while the momentum scale becomes larger. In the right plot, we fix momentum scale to be $2 \mathrm{Gev}$ and plot $C$ as a function of the charm quark mass. When the charm quark mass get smaller, the remaining short distance effect becomes smaller. In Table 2, we show the remaining short distance effect at different charm quark masses. We conclude that the remaining short distance effects are so small that we can neglect them in this work.

\section{Conclusion}

We performed a numerical study of the long distance part of the $K_{L}-K_{S}$ mass difference. The short distance part can be reduce by the inclusion of a valence charm quark. The exponentially increasing term can be identified and subtracted. The remaining short distance effect can be computed by using RI/SMOM techniques and removed from the results. 

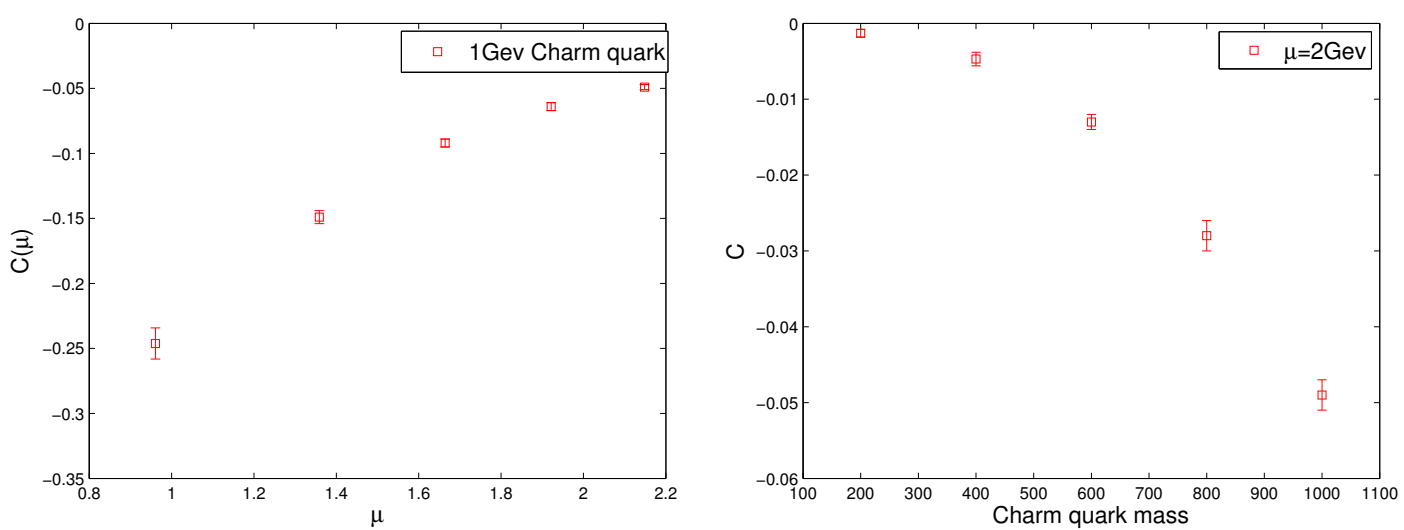

Figure 8: The left plot shows the conversion factor $C(\mu)$ as a function of momentum scale with a $1 \mathrm{Gev}$ charm quark. The right plot shows $C(\mu)$ at different charm quark masses at fixed $\mu=2 \mathrm{Gev}$.

Table 2: Mass differences at different valence charm quark masses (top row) compared with the short distance contribution (bottom row).

\begin{tabular}{cccccc}
\hline$M_{c}(\mathrm{Mev})$ & 200 & 400 & 600 & 800 & 1000 \\
\hline$\Delta M_{K}$ & $0.0440(10)$ & $0.0455(12)$ & $0.0496(13)$ & $0.0556(14)$ & $0.0628(15)$ \\
\hline$\left(\Delta M_{K}\right)_{S D}$ & $6.2 \mathrm{e}-5$ & $2.4 \mathrm{e}-5$ & $6.2 \mathrm{e}-4$ & 0.0013 & 0.0023 \\
\hline
\end{tabular}

The author thanks all his RBC/UKQCD collaborators for discussions and suggestions. Especially thanks to Prof. Norman Chris for detailed instructions and discussion.

\section{References}

[1] S. Herrlich and U. Nierste, Nucl.Phys. B419, 292 (1994), hep-ph/9310311.

[2] G. Buchalla, A. J. Buras, and M. E. Lautenbacher, Rev.Mod.Phys. 68, 1125 (1996), hep-ph/9512380.

[3] RBC and UKQCD Collaborations, N. H. Christ, PoS LATTICE2010, 300 (2010).

[4] RBC Collaboration, T. Blum et al., Phys.Rev. D68, 114506 (2003), hep-lat/0110075. 